\title{
Impacto de la migración parental internacional en el estado nutricional de niños y niñas de 5 a 9 años de la Unidad Educativa Guapán, Cantón Azogues, Provincia del Cañar.
}

\section{Impact of international parental migration on the nutritional status of children aged 5 to 9 years of the Educational Unit Guapán, Azogues Canton, Cañar Province.}

\author{
Aguirre Quezada, Maria Alexandra \\ Universidad Católica de Cuenca, Ecuador \\ maaguirreq@ucacue.edu.ec
}

\begin{abstract}
Resumen
El presente estudio tuvo como objetivo fundamental conocer el impacto de la migración parental internacional sobre el estado nutricional de los niños de 5 a 9 años, de la Unidad Educativa Guapán, en el cantón Azogues. Es un estudio descriptivo de corte transversal en el cual participaron 60 niños hijos de padres migrantes entre 5 y 9 años de edad (28 niños y 32 niñas). Entre los resultados obtenidos se puede destacar que la totalidad de niños y niñas participantes consumen una dieta con una baja presencia de frutas, vegetales y leguminosas que siendo fuentes importantes de vitaminas y minerales sugiere riesgos de déficit de nutrientes específicos, lo que pudiera influir en la presencia de enfermedades. Existe un $60 \%$ de prevalencia de anemia ferropénica en la población estudiada. Se concluyó que los niños con padres migrantes representan un grupo de alto riesgo, por los niveles de malnutrición y alta prevalencia de anemia. Con los resultados de esta investigación se espera aportar con información relevante para que el Distrito Escolar en función de esta problemática proponga al Ministerio de Educación la ejecución de políticas públicas en relación a la migración parental internacional, y atenuar el impacto que puedan tener en la salud y nutrición infantil.
\end{abstract}

Palabras clave: Estado nutricional, Migración parental, Anemia, Calidad de Dieta, Hábitos Alimentarios.

\begin{abstract}
The purpose of this research study is to know the impact of international parental migration on the nutritional status of children aged 5 to 9 years, from the Guapán Educational Unit, canton Azogues. A cross-sectional descriptive study involved 60 children of migrant parents between 5 and 9 years of age (28 children and 32 girls). Among the results obtained, it is possible to emphasize that all the children participating in the diet consume a diet with a low presence of fruits, vegetables and legumes that being important sources of vitamins and minerals suggests risks of specific nutrient deficits that can influence the presence of diseases. There is a $60 \%$ prevalence of iron deficiency anemia in the study population. It was concluded that children with migrant parents represent a high risk group, due to the levels of malnutrition and high prevalence of anemia. The results of this research are expected to contribute with relevant information so that the School District in the light of this problem proposes to the Ministry of Education the implementation of public policies in relation to international parental migration that mitigates the impact they may have on health and child nutrition.
\end{abstract}

Key words: Nutritional status, Parental Migration, Anemia, Quality of diet, Eating habits.

\section{Introducción}

Los movimientos migratorios estimulan cambios con difíciles implicaciones familiares, tanto para los que se van como para los que permanecen en el país de origen.

En la provincia del Cañar, el fenómeno migratorio se agudizó en la década de los años '90, el desempleo y los altos costos de vida ocasionaron que dentro de las familias uno o ambos padres tomen la decisión de migrar a otro país con la intención de incrementar los ingresos económicos para proporcionar un mejor bienestar en educación, salud y vivienda a sus hijos.

Las estadísticas publicadas por el Instituto Nacional Ecuatoriano de Estadísticas y Censos (INEC) confirman que la mayoría de los estudios están focalizados en las remesas que los emigrantes envían, existiendo información 
escasa sobre las consecuencias en la salud y nutrición de los niños que se quedan en el país de origen. ${ }^{1}$

La niñez corresponde al período conocido como "dependencia fundada en unas características biológicas y psíquicas que impiden comportarse como adultos", los padres durante este período del ciclo vital son los responsables del sustento y supervivencia de sus hijos, mientras que en la adolescencia existe una "situación de dependencia que no se deriva ya de incapacidades biológicas o psíquicas sino económicas y sociales". ${ }^{2}$

El proceso migratorio de uno o de los dos padres no solo desata la disgregación de la familia como base de la sociedad sino que se está encomendando el ejercicio de la paternidad a un tercero, provocando el debilitamiento de la responsabilidad de los padres que se asume desde el momento mismo de la concepción.

La migración de los progenitores tiene como consecuencia el enfrentamiento de una serie de cambios trascendentales en el estilo de vida tanto para los que se van como para los que se quedan, en cuanto a relaciones, comunicación, hábitos, comportamientos, alimentación, entre otros. Para un niño, niña o adolescente que experimenta la partida de su padre/madre o de los dos, produce un gran impacto que pone a prueba su capacidad de afrontamiento frente a una secuencia de pérdidas. $^{2}$

La pérdida de vínculos afectivos aumenta la posibilidad de no recibir el mismo cuidado en cuanto a salud, alimentación y tampoco tengan la protección adecuada para evitar todas las formas de violencia a las cuales pueden estar expuestos. $^{2}$

El hijo de padres emigrantes se encuentra inmerso un ambiente donde en ocasiones no es posible el cuidado, una alimentación adecuada y la seguridad. Esto se puede generar por varias razones, una de ellas es el período de crisis económica por el que tiene que pasar la familia mientras que los progenitores logran adaptarse y tener una estabilidad laboral que les permita empezar a enviar dinero. La otra razón es que los cuidadores o personas que quedan a cargo de los niños no les brindan la atención, ni los cuidados que ellos necesitan independientemente de la edad en la que se encuentren, lo cual contribuye a que se desencadene la desnutrición, accidentes en el hogar, consumo de sustancias psicoactivas y problemas del desarrollo. ${ }^{2}$

La información que existe en la provincia Cañar sobre migración internacional indica que el $6 \%$ de su población decidió trasladarse hacia otro país representando un total de 13.554 habitantes. ${ }^{1}$

En Ecuador existen pocos estudios que reflejan la actual situación nutricional y de salud de niños hijos de padres migrantes, y su innegable impacto social que lo convierte en tema prioritario, razón por la cual, los resultados de este estudio serán una guía sobre la cual los actores locales en los que se incluyen las autoridades del Distrito de Salud de Azogues y la Unidad Académica de Salud y Bienestar de la Universidad local, puedan llegar a acuerdos para crear programas preventivos en post de mejorar la calidad de vida de este sector vulnerable, especialmente porque en esta ciudad perteneciente a la provincia del Cañar el fenómeno migratorio internacional según cifras reportadas por el INEC, 3.947 personas del cantón Azogues durante el año 2010 se vieron obligadas a migrar en busca de mejores oportunidades laborales y de vida y que en general los hijos que quedan son de 1 a 12 años de edad, los cuales están al cuidado de un familiar y una condición que no está visible de esta compleja situación, los efectos sobre la nutrición de los niños y niñas que se quedan en el país.

A partir de lo anterior se hace evidente la falta de programas de apoyo y de estudios enfocados en la situación de salud que viven los hogares fragmentados y los niños abandonados privados del vínculo parental, por lo que se torna necesario visibilizar un efecto adicional de la migración.

La relevancia del presente estudio está en su contribución a la comprensión de la migración sobre el estado nutricional de los niños y niñas, y en poner sobre la mesa un importante tema de reflexión y discusión para los organismo estatales y ONG's que trabajan de forma permanente por el cumplimiento de los derechos de los niños, en especial de aquellos que por situaciones de migración parenteral estos derechos se ven vulnerados.

En este contexto, la presente investigación aborda este significativo tema con el objetivo de conocer el impacto de la migración parental internacional sobre el estado nutricional de los niños de 5 a 9 años, de la Unidad Educativa Guapán, cantón Azogues.

\section{Marco Teórico}

En los estudios de Salcedo Rocha que relacionan la migración de los hogares pobres de comunidades rurales e indígenas del Sur de la república mexicana, sobre todo de hombres y a veces de familias enteras que se instalan en los nuevos mercados de trabajo agrícola comercial del norte del país, indican que la migración es un factor de riesgo para la desnutrición de preescolares, debido principalmente a que los primeros meses de ausencia del jefe de hogar, se descapitalizan los hogares de por sí ya pobres, que asociado a la falta de ingresos provenientes del padre, ahora también se ven endeudados con préstamos locales para financiar los desplazamientos de los migrantes. ${ }^{3}$

Una investigación similar realizada por Nuñez - Rocha y colaboradores, muestra que la prevalencia de desnutrición de acuerdo con el indicador talla para la edad, se incrementó casi en el doble en niños pertenecientes a familias migrantes, lo cual es similar a los hallazgos de quién registra una prevalencia de $68 \%$ en migrantes de Jalisco. ${ }^{4}$

Uno de los aspectos de relevancia constituye la influencia de la migración en la morbilidad orgánico-psíquica en niños. En una investigación sobre este aspecto realizada por autores ecuatorianos P. PAREDES, L. BRAVO, A. CALLE realizado en una población de 100 niños y niñas en el Hospital Municipal "Nuestra Señora de la Merced" de la ciudad de Ambato, durante el período comprendido 
entre marzo de 2003-marzo 2004, determinaron que las enfermedades respiratorias y diarreicas constituyen la patología prevalente $(58 \%)$, pero existe también eruptivas, infecciosas en un $12 \%, 8 \%$ de maltrato físico y $12 \%$ de niños sanos. $^{5}$

El $17 \%$ de casos tenían historia de abuso sexual, el $36 \%$ de niños y niñas presentaban disminución del crecimiento y desnutrición de diversos grados. En el diagnóstico psicológico se destaca conducta agresiva $(24,2 \%)$, ansiedad $(10 \%)$, depresión (16\%), enuresis, trastornos del aprendizaje $(8 \%)$. En general existe alteraciones del desarrollo psicomotor $(16 \%)$, violencia intrafamiliar (18\%), y consumo de drogas $(8 \%)$. El bajo rendimiento escolar fue la regla. ${ }^{5}$

En este estudio se estableció que el abandono genera un fenómeno en cadena: trastornos psicológicos, bajo rendimiento escolar, violencia, abuso sexual, así como condiciones de morbilidad, las mismas que son predominantemente infecciosas, y relacionadas con el crecimiento. ${ }^{5}$

Una de las aportaciones de gran relevancia constituye el estudio sobre "Niñez y Migración en el Cantón Cañar", realizada por el Observatorio de los Derechos de la Niñez y Adolescencia en el año 2008, con el propósito de ampliar y fortalecer el conocimiento de las dinámicas y realidades migratorias del Cantón Cañar, pero sobre todo en entender esta realidad a través de las opiniones y percepciones de quienes directa e indirectamente viven sus consecuencias, en contraste con las familias que no lo son. ${ }^{6}$

Los datos recabados en esta investigación permitieron identificar además de las debilidades en el cumplimiento de los Derechos de la Niñez y Adolescencia del Cantón, cinco consecuencias de la migración en los ámbitos de educación, salud, protección social, generación de oportunidades de trabajo para las familias y riesgos de los padres frente al proyecto migratorio. ${ }^{6}$

Riesgos que no son solo para los que se van sino para las familias que se quedan, donde no es suficiente el marco de protección legal, sino el formular y ejecutar políticas "transversales"; pero que requieren cambios profundos en la organización de Estado, recursos humanos capacitados, creación de sistemas de información para el seguimiento y evaluación de las acciones y finalmente un sistema de contraloría social. ${ }^{6}$

En definitiva existen posiciones contrapuestas, por un lado investigaciones que señalan que estos problemas han estado presentes antes de que la migración adquiera las características masivas que tiene ahora y por tanto no deberían relacionarse necesariamente con la migración de los padres. Por otra, evidencias que indican relación morbilidadmigración.

\section{Metodología}

\subsection{Diseño de la investigación}

El tipo de estudio correspondió a una investigación descriptiva, de corte transversal.

\subsection{Población de estudio}

Se realizó en colaboración y bajo el auspicio de la Unidad Educativa Guapán, del cantón Azogues, de la provincia del Cañar en Ecuador; a la fecha de la recolección de información se identificó a 60 hijos de padres migrantes, 28 niños y 32 niñas entre las edades de 5 a 9 años.

En el estudio se incluyeron a todos niños de cinco a nueve años que asisten a esa institución educativa y que viven migración parental internacional de uno o de los dos progenitores y al mismo tiempo quienes contaban con la respectiva autorización de sus padres o tutores para ser partícipes del estudio y firmaron el consentimiento informado, pero se excluyeron a todos los niños de 5 a 9 años que no vivan con sus padres por otro motivo que no sea la migración internacional. Adicionalmente se incluyó a un representante por cada niño.

\subsection{Instrumentos de medición}

Para realizar la recolección de la información se diseñó un cuestionario que incluyó categorías correspondientes a características demográficas y socioeconómicas, evento migratorio, calidad de la dieta y hábitos alimentarios.

Paralelamente se efectuó una ficha de registro de medidas antropométricas de peso, talla y circunferencia de brazo; las mismas se realizaron en los niños con el mínimo de ropa siguiendo el protocolo del Ministerio de Salud Pública del Ecuador ${ }^{7}$ y los datos se analizaron con el software Anthro Plus de la Organización Mundial de la Salud. ${ }^{8}$

\subsection{Definición y tratamiento de las variables}

La variable condiciones socioeconómicas se estudió a través del nivel instrucción de la madre o cuidador, características de la vivienda y ocupación del jefe de hogar. El evento migratorio midió el familiar migrante padre, madre o los dos progenitores, tipo de familia en la que vive el niño/a, familiar a cargo, lugar y tiempo de migración. Se determinó el estado nutricional a través de la evaluación del Índice de Masa Corporal/edad, utilizando los siguientes puntos de corte: delgadez severa menos de 3 desviación típica (DE), delgadez menos de 2DE, normopeso de 2 a menos 2DE, sobrepeso mayor a 2DE y obesidad mayor de 3DE. La circunferencia de brazo correspondió a la reserva proteico energética definiéndose valores mayores a $14.5 \mathrm{~cm}$, 12.5 a 14.5 normal y menor de $12.5 \mathrm{~cm}$ desnutrición. La variable calidad de dieta y hábitos alimentarios se valoró a partir del cuestionario de frecuencia de consumo a través de la escala muy frecuente si el consumo es más de dos veces o una vez por día, frecuente dos o tres veces por semana, poco frecuente una vez a la semana, ocasional una vez por mes y nunca. Para evaluar la calidad de la dieta se utilizó el cuestionario de tamizaje creado por Block y colaboradores, quienes realizaron una encuesta de frecuencia de consumo simplificada en la que incluyeron 13 alimentos que representan la mayor ingesta de grasa en las dietas para desarrollar un tamizador de grasas. ${ }^{9}$ La correlación entre el índice de grasa derivado de esas 13 preguntas y 
el consumo de grasas obtenido de registros múltiples fue similar a la correlación obtenida utilizando cuestionarios más completos de frecuencia de consumo de alimentos, que consideró puntajes de igual o mayor a 30 puntos óptimo consumo de frutas y vegetales, 20 - 29 puntos incluir más frutas, vegetales y granos completos y menos de 20 puntos dieta baja en nutrientes.

\subsection{Métodos para el análisis estadístico}

Para el procesamiento de la información se utilizó el programa SSPS versión 21.

Se llevó a cabo un análisis de correlaciones para determinar el impacto de la migración en el estado nutricional de los niños y niñas investigadas según grupos de edad y progenitor migrante.

La relación entre las variables de estado nutricional, calidad de la dieta, familiar migrante y anemia se estudiaron a través de la prueba Chi - Cuadrado y se consideró significativo un $\mathrm{p}<0.05$.

\section{Resultados}

Los resultados se presentan en un análisis univariado y luego la asociación de variables, como se describe a continuación.

Tabla 1. Nivel de instrucción de la madre o cuidador

\begin{tabular}{ccc}
\hline $\begin{array}{c}\text { Nivel de instrucción de } \\
\text { la madre o cuidador }\end{array}$ & $\mathrm{N}$ & Porcentaje \\
\hline Superior & 23 & 38.3 \\
\hline $\begin{array}{c}\text { De 4 a 6 años de } \\
\text { secundaria }\end{array}$ & 7 & 11.7 \\
\hline $\begin{array}{c}\text { De 1 a 3 años de } \\
\text { secundaria }\end{array}$ & 10 & 16.7 \\
\hline $\begin{array}{c}\text { De 4 a 5 años de } \\
\text { primaria }\end{array}$ & 12 & 20 \\
\hline Ninguna & 8 & 13.3 \\
\hline Total & 60 & 100 \\
\hline
\end{tabular}

Las condiciones socioeconómicas se presentan en las tablas 1, 2 y 3, identificándose que el nivel de instrucción de la madre o del cuidador, corresponde al $38.3 \%$ para estudios superiores, seguido del $20 \%$ de 4 a 5 años de primaria, el $16.7 \%$ de 1 a 3 años de secundaria, el $11.7 \%$ para 4 a 6 años de secundaria y el $13.3 \%$ no disponen de formación, considerando que mientras mayor sea la preparación académica de la persona a cargo del niño/a mejor preparados están para la crianza de los mismos.

Tabla 2. Riesgo de la vivienda

\begin{tabular}{ccc}
\hline Riesgo de la vivienda & $\mathrm{N}$ & Porcentaje \\
\hline Vivienda de bajo riesgo & 4 & 6,7 \\
\hline $\begin{array}{c}\text { Vivienda de mediano } \\
\text { riesgo }\end{array}$ & 43 & 71,7 \\
\hline Vivienda de alto riesgo & 13 & 21,6 \\
\hline Total & 60 & 100 \\
\hline
\end{tabular}

El $21.7 \%$ habitan en infraestructuras de alto riesgo. Las familias estudiadas pertenecen al estrato tres popular alto, en función de las actividades labores a las que acceden el jefe de hogar.

Tabla 3. Nivel de clase social del jefe de hogar

\begin{tabular}{ccc}
\hline $\begin{array}{c}\text { Nivel de clase social } \\
\text { del jefe de hogar }\end{array}$ & $\mathrm{N}$ & Porcentaje \\
\hline Estrato medio alto & 17 & 28.3 \\
\hline Estrato medio & 14 & 23.3 \\
\hline Estrato popular alto & 18 & 30 \\
\hline Estrato popular bajo & 11 & 18.3 \\
\hline Total & 60 & 100 \\
\hline
\end{tabular}

El resumen del país de residencia de los padres migrantes, se exponen en la tabla 4, observando que el principal es Estados Unidos con un $85.1 \%$, seguido de España con el $8.3 \%$ y se observa igual representación para Italia y otros destinos con el $3.3 \%$.

Esta situación confirma que el destino migratorio mayor es Estados Unidos que es en gran parte la realidad migratoria del cantón Azogues.

Tabla 4. Familiar migrante y lugar de la migración

\begin{tabular}{|c|c|c|c|c|c|c|c|c|c|}
\hline \multirow{3}{*}{$\begin{array}{l}\text { Familiar } \\
\text { migrante }\end{array}$} & \multicolumn{8}{|c|}{ Lugar de la mioración } & \multirow{3}{*}{$\begin{array}{c}\text { Tota } \\
\% \\
\end{array}$} \\
\hline & \multicolumn{2}{|c|}{$\begin{array}{l}\text { Estados } \\
\text { Unidos }\end{array}$} & \multicolumn{2}{|c|}{ España } & \multicolumn{2}{|c|}{ Italia } & \multicolumn{2}{|c|}{ Otro } & \\
\hline & $\mathrm{N}$ & $\%$ & $\mathrm{n}$ & $\%$ & $\mathrm{n}$ & $\%$ & $\mathrm{~N}$ & $\%$ & \\
\hline Padre & 44 & 73,4 & 2 & 3,3 & 2 & 3,3 & 0 & 0 & 80 \\
\hline Madre & 4 & 6,7 & 1 & 1,7 & 0 & 0 & 1 & 1,7 & 10 \\
\hline $\begin{array}{l}\text { Padre y } \\
\text { madre }\end{array}$ & 3 & 5 & 2 & 3,3 & 0 & 0 & 1 & 1,7 & 10 \\
\hline Total & 51 & 85,1 & 5 & 8.3 & 2 & 3.3 & 2 & 3.3 & 100 \\
\hline
\end{tabular}

En relación al tiempo de migración de los progenitores, los porcentajes se encuentran detallados en la tabla 5, resaltando que el $45 \%$ de los progenitores se encuentre fuera del país por menos de un año.

Tabla 5. Familiar migrante y tiempo de migración

\begin{tabular}{|c|c|c|c|c|c|c|c|}
\hline \multirow{3}{*}{$\begin{array}{l}\text { Familiar } \\
\text { migrante }\end{array}$} & \multicolumn{6}{|c|}{ Tiempo de migración de progenitores } & \multirow{2}{*}{$\begin{array}{c}\text { Total } \\
\%\end{array}$} \\
\hline & \multicolumn{2}{|c|}{$\begin{array}{c}\text { Menos de } \\
\text { un año }\end{array}$} & \multicolumn{2}{|c|}{$\begin{array}{l}1 \text { a } 4 \\
\text { años }\end{array}$} & \multicolumn{2}{|c|}{$\begin{array}{l}\text { Más de } 5 \\
\text { años }\end{array}$} & \\
\hline & $\mathrm{N}$ & $\%$ & $\mathrm{n}$ & $\%$ & $\mathrm{~N}$ & $\%$ & \\
\hline \multirow[t]{3}{*}{ Padre } & 1 & 3 & 1 & 1 & 1 & 30 & 80 \\
\hline & 9 & 1. & 1 & 8. & 8 & & \\
\hline & & 7 & & 3 & & & \\
\hline Madre & 5 & $\begin{array}{l}8 . \\
3\end{array}$ & 1 & $\begin{array}{l}1, \\
7\end{array}$ & 0 & 0 & 10 \\
\hline $\begin{array}{l}\text { Padre y } \\
\text { madre }\end{array}$ & 3 & 5 & 2 & $\begin{array}{l}3, \\
3\end{array}$ & 1 & $\begin{array}{l}1 . \\
7\end{array}$ & 10 \\
\hline \multirow[t]{2}{*}{ Total } & 2 & 4 & 1 & 2 & 1 & 31 & 100 \\
\hline & 7 & 5 & 4 & $\begin{array}{l}3, \\
3\end{array}$ & 9 & .7 & \\
\hline
\end{tabular}

$\mathrm{Al}$ investigar sobre la persona a cargo del niño o niña, se puede observar que en la mayoría de casos la persona a cargo es la madre, en un $78.3 \%$; lo que constituye un aspecto positivo en la crianza del niño, el vivir con los tíos o los abuelos es una demostración del cambio en la dinámica familiar que la migración trae como consecuencia, información descrita en la tabla 6.

En la tabla 7, se resume la prevalencia de malnutrición en la población estudiada según indicadores antropométricos, 
Tabla 6. Familiar a cargo

\begin{tabular}{ccc}
\hline Familiar a cargo & $\mathrm{N}$ & Porcentaje \\
\hline Mamá & 48 & 78.3 \\
\hline Papá & 6 & 6.7 \\
\hline Hermano/a mayor & 1 & 1.7 \\
\hline Abuelos maternos & 4 & 6.7 \\
\hline Abuelos paternos & 1 & 1.7 \\
\hline Tíos & 1 & 1.7 \\
\hline Total & 60 & 100 \\
\hline
\end{tabular}

en el grupo de estudio el $8.3 \%$ tienen un IMC bajo, es decir, el peso está por debajo de lo que su talla les permite y al relacionarlos el resultado es delgadez, pero en razón de que el peso es una medida de fácil recuperación los niños con un adecuado seguimiento podría armonizar el peso para su talla. En cuanto a sobrepeso y obesidad se encuentran el $10 \%$ de los niños con el consiguiente riesgo para su salud. El retardo en el crecimiento medido con el indicador talla/edad es uno de los aspectos más destacados de la antropometría, y por ello, puede tener importancia social. En el grupo en estudio el $28.4 \%$ presenta retardo en su crecimiento, lo que es un problema importante pues de un grupo pequeño en estudio (60 niños) 17 presentan retardo que puede ser un indicio de un proceso de desnutrición crónica con un historial nutricional de largo plazo que amerita identificar para definir las causas. Las reservas proteico energéticas disminuidas se encuentra el $11.7 \%$ compatible con malnutrición leve o moderada.

Tabla 7. Prevalencia de malnutrición en la población estudiada según tipo de indicador

\begin{tabular}{c|c|c|c}
\hline \multicolumn{2}{c|}{ INDICADOR } & n & Porcentaje \\
\hline Índice de & Sobrepeso & 3 & 5 \\
\cline { 2 - 4 } $\begin{array}{c}\text { masa } \\
\text { Corporal } \\
\text { IMC }\end{array}$ & Obesidad & 3 & 5 \\
\cline { 2 - 4 } & Déficit & 5 & 8.3 \\
\hline \multicolumn{2}{c|}{ Retardo en el crecimiento } & 17 & 28.4 \\
\hline \multicolumn{2}{c}{$\begin{array}{c}\text { Reservas proteico energéticas } \\
\text { disminuidas }\end{array}$} & 7 & 11.7 \\
\hline
\end{tabular}

El $60 \%$ de los niños tienen valores de hemoglobina bajo $11 \mathrm{mg} / \mathrm{dl}$ que es el punto de corte para determinar anemia a esta edad (OMS, 2001), además es mayor en los niños que en las niñas y el grupo de edad más afectado es el de 7 a 9 años, información referida en la tabla 8.

Tabla 8. Hemoglobina corregida por altitud y sexo

\begin{tabular}{lccccc}
\hline $\begin{array}{l}\text { Hemoglobina } \\
\text { corregida por } \\
\text { altitud }\end{array}$ & \multicolumn{2}{c}{ Masculino } & \multicolumn{2}{c}{ Femenino } & Total \\
\cline { 2 - 6 } & $\mathrm{N}$ & $\%$ & $\mathrm{~N}$ & $\%$ & \\
\hline Anemia & 15 & 33.3 & 12 & 26.7 & 27 \\
\hline $\begin{array}{l}\text { Valor normal para } \\
\text { la edad }\end{array}$ & 5 & 11.1 & 13 & 28.9 & 18 \\
\hline \multicolumn{1}{c}{ Total } & 20 & 44.4 & 25 & 55.6 & 45 \\
\hline
\end{tabular}

La tabla 9 resume la relación entre el IMC con el familiar migrante y se obtuvo un valor de $\mathrm{p}$ de 0.021 , confirmando que hay una relación estadísticamente significativa, indicando que la ausencia de la madre en el hogar muestra mayor desventaja dentro de un proceso migratorio pues el $50 \%$ de niños con delgadez viven esta situación, a diferencia del otro grupo en el que están el padre cuyo caso la prevalencia es del $4.2 \%$.

Tabla 9. IMC/ edad en relación al familiar migrante

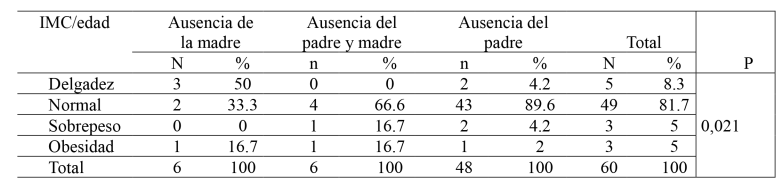

Al relacionar la hemoglobina corregida por altitud en relación al progenitor migrante, se dispone un valor de $\mathrm{p}$ de 0.301 , probando que no hay diferencia respecto a la ausencia del padre o la madre en cuanto a la anemia, información anotada en la tabla 10.

Tabla 10. Hemoglobina corregida por altitud en relación al progenitor migrante

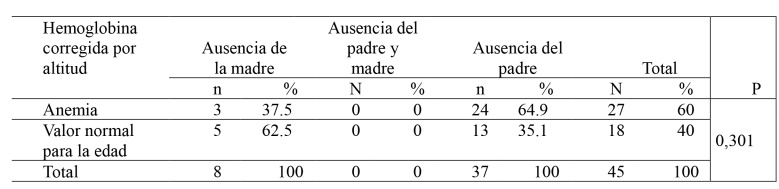

En la tabla 11 se consolida la información relacionada a hábitos alimentarios en el grupo de 5 a 6 años 11 meses 29 días, en la que se tiene que el grupo de alimentos de consumo muy frecuente son los cereales y derivados, especialmente el arroz que es alimento de consumo cotidiano, a diferencia del consumo frecuente que corresponde al grupo del azúcar, en la categoría poco frecuente predomina el consumo de carnes y derivados que hace suponer que los niños y niñas investigados podrían presentar un déficit de proteínas de buena calidad para cubrir sus necesidades de proteína y hierro. Otra particularidad que se observa es el alto porcentaje de consumo poco frecuente en relación a lácteos, considerando que este alimento es fuente de calcio tan necesario en esta etapa del ciclo de vida en la que se encuentran los niños investigados.

En la opción eventual se identificó una baja ingesta de pescados y mariscos posiblemente debido a la región en la que vive el grupo en estudio (sierra), cultura alimentaria, costo y oferta de estos productos.

Se resalta el hecho del consumo muy frecuente de tubérculos referido especialmente a la papa, alimento de consumo diario en la sierra y un soporte nutricional importante dentro del patrón alimentario por sus diferentes variedades y formas de preparación infaltable en el menú diario ya sea por su amplia disponibilidad como por su bajo costo e incluso de autoconsumo en los sectores rurales.

El patrón alimentario de los niños en función de la frecuencia de su consumo son: cereales, tubérculos, azúcar, aceites y grasa y bebidas no lácteas. Este aspecto demuestra que la dieta de estos niños es carente de nutrientes muy importantes, macro y micronutrientes. 
Tabla 11. Hábitos alimentarios en el grupo de 5 a 6 años 11 meses 29 días

\begin{tabular}{cccccc}
\hline \multirow{2}{*}{$\begin{array}{c}\text { Grupo de } \\
\text { alimentos }\end{array}$} & $\begin{array}{c}\text { Muy } \\
\text { frecuente }\end{array}$ & Frecuente & $\begin{array}{c}\text { Poco } \\
\text { frecuente }\end{array}$ & Eventual & Nunca \\
\hline $\begin{array}{c}\text { Cereales y } \\
\text { derivados }\end{array}$ & 26,7 & 10 & 3,3 & 1,7 & 0 \\
\hline Lácteos & 3.0 & 8.0 & 30.7 & 0 & 0 \\
\hline $\begin{array}{c}\text { Huevos y } \\
\text { derivados }\end{array}$ & 3,3 & 8,4 & 25 & 5 & 0 \\
\hline $\begin{array}{c}\text { Carnes } \\
\text { derivados }\end{array}$ & 3,3 & 5 & 33,4 & 0 & 0 \\
\hline $\begin{array}{c}\text { Pescados y } \\
\text { mariscos }\end{array}$ & 0 & 1,7 & 11,7 & 20 & 8,3 \\
\hline Vegetales & 5 & 6,7 & 20 & 10 & 0 \\
\hline Tubérculos & 28 & 10 & 3.7 & 0 & 0 \\
\hline Frutas frescas & 15 & 8,3 & 16,7 & 1,7 & 0 \\
\hline Frutos secos & 0 & 0 & 5 & 6,7 & 30 \\
\hline $\begin{array}{c}\text { Aceites y } \\
\text { grasas }\end{array}$ & 11,7 & 5 & 23,3 & 0 & 1,7 \\
\hline Azúcar & 18,3 & 16,7 & 6,7 & 0 & 0 \\
\hline $\begin{array}{c}\text { Chocolate y } \\
\text { bollería }\end{array}$ & 6,7 & 3,3 & 16,7 & 15 & 0 \\
\hline $\begin{array}{c}\text { Bebidas no } \\
\text { lácteos }\end{array}$ & 11,7 & 5 & 13,3 & 11,7 & 0 \\
\hline
\end{tabular}

Tabla 12. Hábitos alimentarios en el grupo de 5 a 6 años 11 meses 29 días

\begin{tabular}{cccccc}
\multirow{2}{*}{$\begin{array}{c}\text { Grupo de } \\
\text { alimentos }\end{array}$} & \multicolumn{5}{c}{ Porcentaje de frecuencia de consumo } \\
\cline { 2 - 6 } & $\begin{array}{c}\text { Muy } \\
\text { frecuente }\end{array}$ & Frecuente & $\begin{array}{c}\text { Poco } \\
\text { frecuente }\end{array}$ & Eventual & Nunca \\
\hline $\begin{array}{c}\text { Cereales y } \\
\text { derivados }\end{array}$ & 40 & 13,3 & 3,3 & 1,7 & 0 \\
\hline Lácteos & 12 & 13,2 & 33,1 & 0 & 0 \\
\hline $\begin{array}{c}\text { Huevos y } \\
\text { derivados }\end{array}$ & 16,7 & 10 & 21,6 & 10 & 0 \\
\hline $\begin{array}{c}\text { Carnes } \\
\text { derivados }\end{array}$ & 13,3 & 10 & 31,7 & 3,3 & 0 \\
\hline $\begin{array}{c}\text { Pescados y } \\
\text { mariscos }\end{array}$ & 1,7 & 1,7 & 13,3 & 25 & 16,6 \\
\hline Vegetales & 5 & 10 & 26,6 & 16,7 & 0 \\
\hline Tubérculos & 36.6 & 15 & 6.7 & 0 & 0 \\
\hline $\begin{array}{c}\text { Frutas } \\
\text { frescas }\end{array}$ & 25 & 8,3 & 20 & 5 & 0 \\
\hline $\begin{array}{c}\text { Frutos } \\
\text { secos }\end{array}$ & 1,7 & 3,3 & 3,3 & 6,7 & 43,3 \\
\hline $\begin{array}{c}\text { Aceites y } \\
\text { grasas }\end{array}$ & 23,3 & 8,4 & 23,3 & 0 & 3,3 \\
\hline Azúcar & 36,6 & 13,3 & 6,7 & 1,7 & 0 \\
\hline $\begin{array}{c}\text { Chocolate y } \\
\text { bollería }\end{array}$ & 8,3 & 15 & 11,7 & 23,3 & 0 \\
\hline $\begin{array}{c}\text { Bebidas } \\
\text { no lácteos }\end{array}$ & 16,7 & 13,3 & 13,3 & 13,3 & 1,7 \\
\hline
\end{tabular}

Al realizar el análisis de los hábitos alimentarios en el grupo de 7 a 9 años se puede observar en la tabla 12, que el grupo de alimentos de consumo muy frecuente son los cereales y derivados, a diferencia del consumo frecuente que corresponden con el mismo porcentaje a los grupos de azúcar y bebidas no lácteas, en la categoría poco frecuente predomina la carne y derivados, en la opción eventual se identificó la ingesta de pescados y mariscos y de manera muy preocupante se afirma en la última opción a los frutos secos.

Tabla 13. Retardo en talla de los niños investigados en relación al progenitor migrante

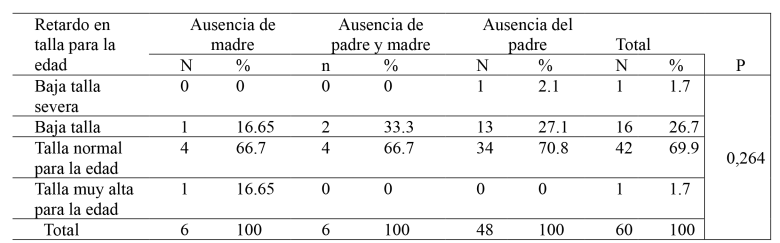

En este estudio al relacionar el progenitor migrante con el retardo en talla (tabla 13), estado de las reservas proteico energéticas (tabla 14) y hemoglobina corregida por altitud en relación al progenitor migrante (tabla 10) se obtuvieron valores de p 0.264 , p 0.148 y p 301, respectivamente, confirmando que no hay una relación estadística significativa.

Tabla 14. Estado de las Reservas proteico Energéticas y su asociación con el progenitor migrante

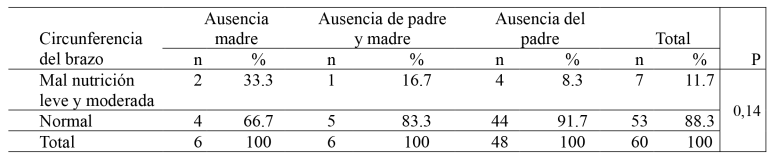

Sin embargo al relacionar el progenitor migrante con la calidad de la dieta en relación a consumo de grasa se obtuvo un valor de p 0.046 , por lo que existe una relación estadística significativa, información descrita en la tabla 15.

Tabla 15. Calidad de la dieta en relación a consumo de grasa y su asociación con el progenitor migrante

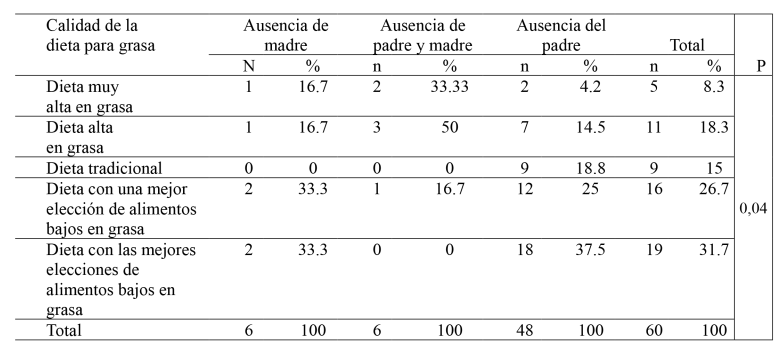

\section{Conclusiones y recomendaciones}

El análisis partió de un modelo causal que considera las variables socioeconómicas de las familias a las que pertenecen los niños investigados, migración, calidad de la dieta y hábitos alimentarios, como variables independientes que actuando a través de las variables que definen el estado nutricional determinan el comportamiento del estado nutricional de los niños.

En este estudio se ha demostrado la prevalencia de malnutrición en la población de estudio. Por lo tanto, debería considerarse la necesidad de diseñar una propuesta para realizar la evaluación nutricional continua en la unidad educativa Guapán.

En el grupo en estudio el $28.4 \%$ presenta retardo en su crecimiento que es un problema importante, pues de un grupo pequeño en estudio (60 niños) 17 presentan retardo que puede ser un indicio de un proceso de desnutrición crónica con un historial nutricional de largo plazo que amerita identificar para definir las causas.

El $100 \%$ de los niños y niñas investigadas consumen una dieta con una baja presencia de frutas, vegetales y leguminosas que siendo fuentes importante de vitaminas y minerales el resultado lógico es una dieta baja en nutrientes importantes que dependiendo de su magnitud e intensidad 
puede influir en la presencia de enfermedades causadas por un desbalance alimentario. Es necesario orientar a los adultos a través de intervenciones de educación nutricional sobre el beneficio del consumo de alimentos saludables, y que configuren patrones alimentarios adecuados que puedan evitar la presencia de enfermedades a futuro.

El presente estudio identificó casos de obesidad, sobrepeso, déficit, retardo en el crecimiento y reservas proteico energéticas disminuidas a edades muy tempranas en la Unidad Educativa Guapán, lo cual evidencia que esta población no está fuera de la realidad nutricional a nivel nacional.

La edad escolar es la etapa ideal en la vida para aplicar medidas con enfoque preventivo para evitar la aparición de enfermedades crónicas en la adultez, es una época de adquisición de hábitos; enseñarlos de manera precoz podría constituir la estrategia para el ejercicio de un estilo de vida saludable.

Existen algunos estudios cuyos resultados no mantienen correspondencia con los de este estudio. Uno de ellos es una investigación realizada en el hospital municipal "Nuestra Señora de la Merced" de la ciudad de Ambato sobre el estado nutricional de hijos de padres migrantes en el que se reportó un $56 \%$ de niños con disminución del crecimiento. ${ }^{5}$

Pese a que se analiza la realidad de familias migrantes, y se pudiese inferir mejores condiciones económicas por el envío de remesas, una quinta parte de la población habitan en infraestructuras de alto riesgo, es decir, inadecuado acceso a servicios básicos y problemas con la infraestructura, en tal virtud se considera un factor crítico que puede incidir en el estado de salud de los niños, estimulando el desarrollo o complicación de enfermedades. Los resultados descritos no mantienen relación con el estudio realizado por en la comunidad de Manzanapata del cantón Cañar en la que el índice de riesgo vivienda corresponde en un $85 \%$ a 10. mediano riesgo y no se identifican niños que habiten en hogares con alto riesgo. ${ }^{10}$

El hecho de que los padres migraron en un tiempo menor a un año, constituye una clara expresión de la calidad de vida de los niños, analizada desde diferentes ópticas que no necesariamente se enfoca a los aspectos materiales, sino en lo fundamental a la seguridad emocional de los niños que al quedar sin sus progenitores se someten al cuidado de otros familiares que de forma muy difícil logran cubrir el amor y la estabilidad emocional garantizada por los padres biológicos.

De mantenerse el hábito en el consumo de dietas altas en grasa la incidencia de diagnósticos de sobrepeso y obesidad se incrementarán predisponiendo a esta población a la posible aparición de enfermedades crónicas en la edad adulta.

La falta de asociación entre el estado nutricional categorizado por antropometría, el retardo en talla y el progenitor migrante canaliza la ejecución de futuras investigaciones en las que se realice modificación a los instrumentos y permitan la medición de estas variables de manera cuantitativa y objetiva.
Los resultados obtenidos pueden ser complementados con futuros estudios para proporcionar una base sólida que orienten de una manera adecuada la práctica de promoción de salud, dando énfasis a los hábitos de alimentación, calidad de dieta y antropometría en hijos de padres migrantes.

\section{Referencias Bibliográficas}

1. INEC. Informe estadístico de migración. Instituto Nacional de Estadísticas y Censos; 2013. Recuperado el 2015, de: http://www.ecuadorencifras.gob.ec/documentos/webinec/ECV/ECV_2015/.

2. Comfenalco Q. Así somos. Húerfanos de padres vivos; 2009. 10-12, Recuperado el 2015.

3. Salcedo A. El proceso migratorio como un factor de riesgo en la desnutrición crónica del niño. Revista de salud pública México. 2008;34:518-522.

4. Georgina Nuñez MByBC. Desnutrición en preescolares de familias migrantes. Revista de salud pública de México. 1998;40(3):248-255. Recuperado de http://www.redalyc.org/pdf/106/10640305.pdf.

5. P Paredes LByAC. Impacto de la migración en la salud infantil; 2004. Boletín de pediatría, 44(189), 137-149.

6. Escobar A. Niñez y migración del cantón Cañar. Unicef; 2008.

7. Manual de Procedimientos de Antropometría y Determinación de la Presión Arterial. Ecuador. Ecuador; 2012.

8. OMS. Estudio multicéntrico sobre el patrón de crecimiento para los niños.; 2008. Organización Mundial de la Salud.

9. Gladys Block MDNMHyMM Carolyn Clifford. A Brief Dietary Screen for High Fat Intake. Journal of $\mathrm{Nu}-$ trition Education and Behavior;21(5):199-207. DOI: http://dx.doi.org/10.1016/S0022-3182(89)80003-2.

0. Tenesaca R. Seguridad alimentaria de la comunidad de Manzanapata y alternativas para la mejora del cantón Cañar; 2010. Universidad de Cuenca.

Recibido: 25 de enero de 2017

Aceptado: 3 de mayo de 2017 
